\title{
Nonlinear broadband photoluminescence of graphene induced by femtosecond laser irradiation
}

\author{
Wei-Tao Liu,1 S. W. Wu,2 P. J. Schuck,2 M. Salmeron,2 Y. R. Shen,1 and F. Wang1,* \\ ${ }_{1}$ Physics Department, University of California at Berkeley, Berkeley, California 94720, USA \\ 2The Molecular Foundry, Lawrence Berkeley National Laboratory, Berkeley, California 94720, USA
}

Upon femtosecond laser irradiation, a bright, broadband photoluminescence is observed from graphene at frequencies well above the excitation frequency. Analyses show that it arises from radiative recombination of a broad distribution of nonequilibrium electrons and holes, generated by rapid scattering between photoexcited carriers within tens of femtoseconds after the optical excitation. Its highly unusual characteristics come from the unique electronic and structural properties of graphene.

Graphene is an $s p 2$-hybridized carbon monolayer that has many uncommon attributes, from exotic electrical ${ }^{1}$ and thermal ${ }^{2}$ transport to extraordinary mechanical properties. ${ }^{3}$ It also exhibits unusual linear optical behavior, which shows a universal absorption constant ${ }^{4}$ and can be effectively controlled by electrical gating. ${ }^{5}$ The nonlinear optical response of graphene could be even more interesting: it is well known that unusual nonlinear optical phenomena can arise from low-dimensional confinement of carriers as has been demonstrated extensively in semiconductor quantum well structures. ${ }^{6}$ Moreover, graphene has a unique band structure that could give rise to extraordinary nonlinear optical properties, yet such aspects have been overlooked so far.

In this Rapid Communication, we report the observation of an unusually bright, broadband nonlinear photoluminescence (PL) generated in graphene upon femtosecond laser irradiation. It has a significant blueshifted component covering the entire visible spectrum when excited by near-infrared photons, and has very different characteristics from, for example, those of two-photon photoluminescence (TPPL) in noble metals. ${ }^{7}$ Our analysis suggests that the graphene nonlinear PL arises from a broad distribution of nonequilibrium electron-hole (e-h) gas created via rapid scattering between a high density of photoexcited carriers. Although this mechanism is not limited to graphene, its two-dimensional (2D) nature and unusual band structure dramatically enhance the PL efficiency and bandwidth.

Our experiments were performed with a $76 \mathrm{MHz}$ Ti:Sapphire oscillator pumping an optical parametric oscillator with $\sim 150$ fs output pulses tunable within $\sim 1.4-2.2 \mathrm{eV}$. As shown schematically in Fig. 1(a)_, the beam was focused on the sample at normal incidence, and the PL was collected in the backscattered direction. The signal was then detected by either a spectrograph equipped with a silicon charge-coupled device for monitoring spectra, or a single-photon counting silicon avalanche photodiode for detecting the integrated signal. The continuous-wave_cw_ Raman spectra were taken with a helium-

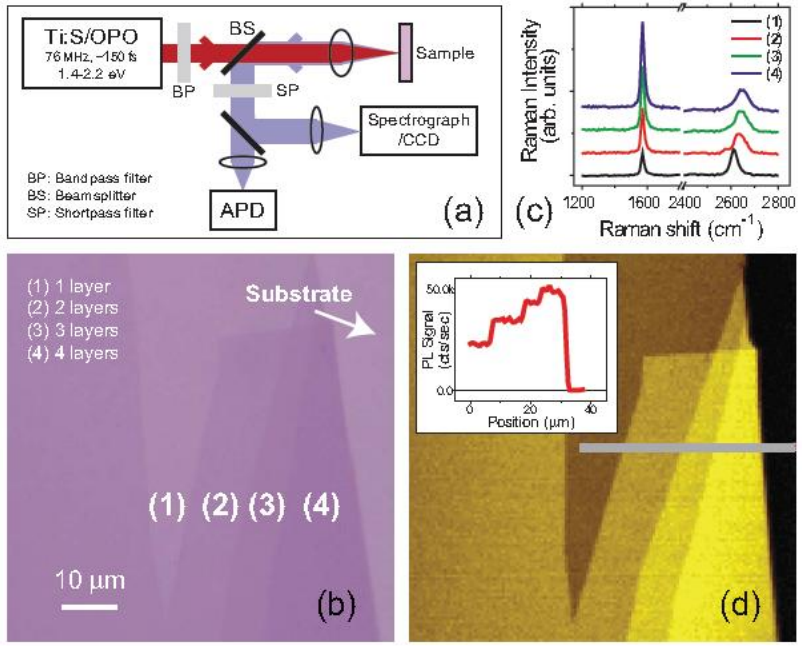

FIG. 1. (Color online) (a) Schematics of the experimental etup. (b) White-light microscopy image of a thin graphene sample. (c) cw Raman spectra taken at positions (1)-(4). (d) Nonlinear PL image of the same sample as excited by $1.5 \mathrm{eV}$ femtosecond ulses with signal integrated from 1.66 to $3.11 \mathrm{eV}$. Inset: PL signal long the line cut. neon laser at $632.8 \mathrm{~nm}$. All measurements were performed in atmosphere at room temperature. Thin graphene layers were prepared via mechanical exfoliation following the standard procedure described in Ref. ${ }^{8}$. Figure 1(b) shows the bright-field optical microscopy image of a graphene sample on silicon covered by a 295-nm-thick $\mathrm{SiO} 2$ layer. cw Raman spectra at different sample positions are displayed in Fig. 1(c). The sample was found to have one to four monolayers in different regions [labeled in Fig. 1(b)], seen from both the optical contrast and relative intensities of different Raman modes, and had a negligible amount of defects as evidenced by the absence of $D$ mode at $\sim 1350 \mathrm{~cm}^{-1}{ }^{9}$ Upon femtosecond laser irradiation, strong PL signals were detected at frequencies both higher and lower than incident photons. Surprisingly, the 

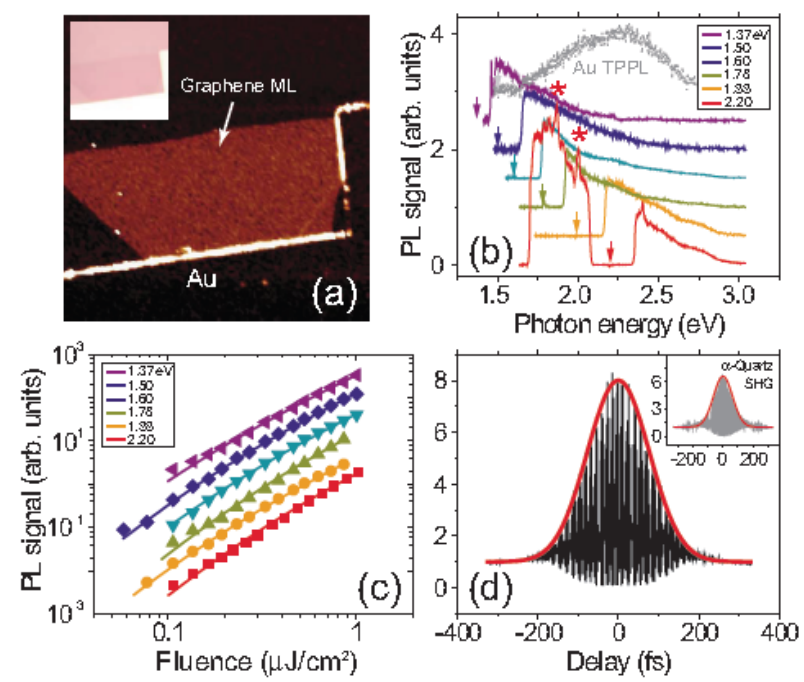

FIG. 2. (Color online) (a) PL image of a graphene monolayer alongside a Au film. Inset: white-light microscopy image of the same area. (b) PL spectra at various excitation frequencies (marked by arrows) from a few-layer graphene sample on glass substrate (sharp cuts are due to interference filters). The $G$ and $2 D$ Stokes Raman modes upon $2.20 \mathrm{eV}$ excitation are marked by “ * ". The Au TPPL spectrum is shown for comparison (gray). (c) Integrated PL signal vs excitation density at various excitation frequencies _curves are vertically offset for clarity_. Solid lines are fits using Eq. (2). (d) PL signal as a function of the delay between two excitation pulses. Red curve (the envelope) is a Gaussian fit. Inset: the $\mathrm{SH}$ autocorrelation trace from a-quartz. The excitation frequency is $1.5 \mathrm{eV}$ and the PL signal is integrated from 1.66 to $3.11 \mathrm{eV}$ in (a) and (d). blushifted (anti-Stokes) component, which is typically negligible in conventional materials, was very bright and had a comparable strength to the redshifted (Stokes) component. We will focus on the blueshifted PL component because it is characteristically unique. Figure 1(d) shows the PL image of the same area in Fig. 1(b) under $1.5 \mathrm{eV}$ excitation, with signal integrated between 1.66 and $3.11 \mathrm{eV}$. The inset displays a line cut of the signal strength across the sample, showing PL increased in steps. The PL strength per atomic sheet decreased considerably in thicker layers, despite that the variation in excitation intensity was small across the ultrathin sample. Remarkably, the blueshifted PL from a graphene monolayer was a few hundred times stronger than the TPPL signal from a 50-nm-thick gold film when compared side-by-side [Fig. 2(a) ]except at the film edge where TPPL experienced giant local-field enhancement ${ }^{7}$. Representative PL spectra are displayed in Fig. 2(b), obtained from samples prepared on a transparent glass substrate to avoid interference effects from oxide-covered silicon substrates. ${ }^{10}$ Again Raman spectra were measured to ensure the sample quality. The redshifted PL upon $2.2 \mathrm{eV}$ excitation is shown for comparison. In all cases, the

blueshifted PL was characterized by a featureless continuum that decreased monotonically toward higher frequency. For a given excitation frequency, the spectral profile remained similar at different thickness and laser fluence. The PL strength had highly nonlinear fluence dependence. Figure 2(c) displays the spectrally integrated PL strength versus the absorbed fluence in a double-logarithm representation, which has a slope changing from $\sim 3$ to 2 with increasing laser fluence.

Dynamic response of the PL to excitation was checked via autocorrelation measurements. ${ }^{11,12}$ The full-width-half-maximum (FWHM) of the PL trace envelope [Fig. 2(d)] is only $\sim 10-20$ fs broader than that of the second-harmonic (SH) signal from $\alpha$ quartz (inset). Since broadening of the PL FWHM relative to the pulse duration roughly equals the intermediate-state lifetime, ${ }^{12}$ it indicates that the PL must arise from an e-h distribution generated within $~ 10-20 \mathrm{fs}$ after the excitation.

We now examine possible mechanisms that give rise to this unusually bright and broadband PL. We note that the observed radiation has typical characteristics of luminescence but not those of the output from coherent nonlinear optical processes. The latter is usually highly directional, and/or its generation requires a sufficiently long interaction length in a medium, which was clearly not the case for a monolayer graphene. The blueshifted PL must originate from radiative recombination of carriers created by either direct multiphoton excitations [schematically illustrated in Fig. 3(a)], or scattering of onephoton-excited carriers [Fig. 3(b)]. TPPL from Au is representative of the former but it exhibits a peak at $\sim 2.2 \mathrm{eV}$ that does not shift with the excitation frequency [Fig. 2(b)], ${ }^{7}$ which is contrary to the graphene PL. Direct multiphoton absorption was also found to be insignificant in graphene. ${ }^{13}$ On the other hand, main features of the graphene PL can be explained by the latter process. The electronic structure of graphene is characterized by linear bands from $\pi$ and $\pi$ orbitals within $\sim 3 \mathrm{eV}$ around the Dirac point. Multilayer samples have similar band structures in the photon energy range of interest. ${ }^{4}$ Upon femtosecond laser irradiation, resonant one-photon excitation at frequency $\omega_{0}$ generates a dense 
population of electrons (holes) at $E_{0}=h \omega_{0} / 2\left(-h \omega_{0} / 2\right)$ in the conduction (valence) band. Subsequently, efficient intraband scattering between photoexcited electrons (holes) ${ }^{13}$ can create a population spanning the entire range from nearly 0 to $2 E_{0}\left(-2 E_{0}\right)$ (marked by broken lines) [Fig. 3(b)]. As the Coulomb scattering rate scales inversely with the exchanged momentum, $|\mathbf{Q}|,{ }^{14}$ it results in a monotonic decay of electron (hole) population toward $2 E_{0}(-2 E 0)$. The e-h recombination can then generate PL signals in a continuous band up to $2 h \omega_{0}$, with diminishing intensity toward higher frequencies.

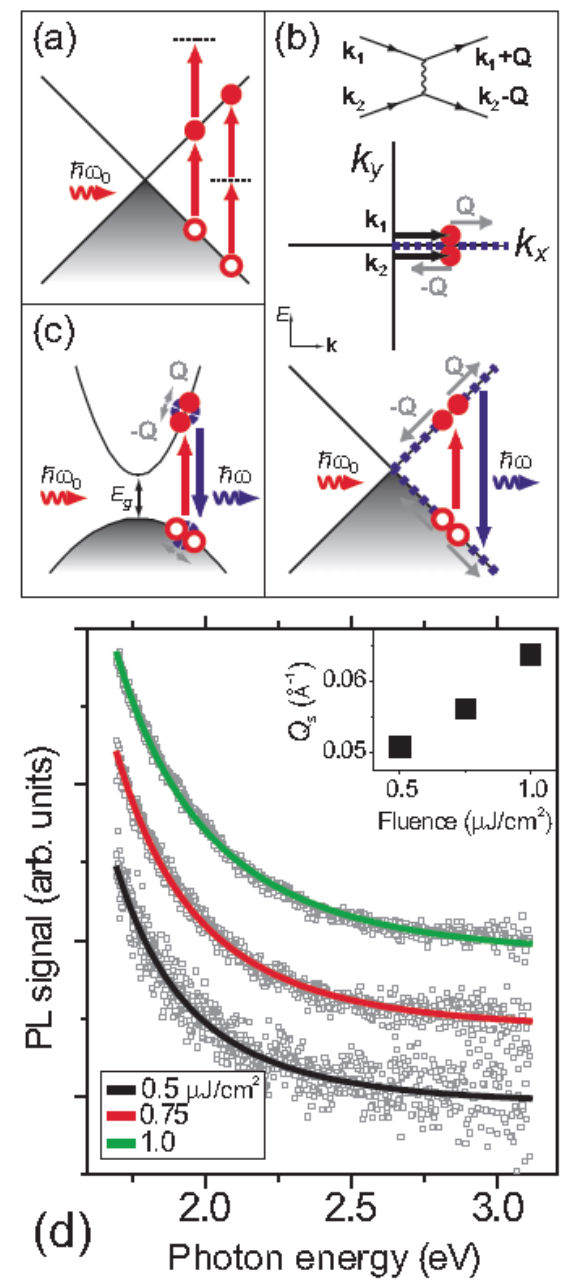

FIG. 3. (Color online) (a) Schematics of multiphoton transitions in graphene near the Dirac point. (b) Schematics of elastic scattering between two carriers with initial momentum $\mathbf{k}$, $\mathbf{k} 2$, and exchanged momentum $\mathbf{Q}$. Upper (lower) panel is the view in $\mathbf{k}(E-\mathbf{k})$ plane, red dots (circles) refer to hot electrons (holes), respectively. The broken blue line is the trajectory of final states allowed by energy and momentum conservation. (c) Schematics of the same process depicted in (b) but in a system of parabolic bands and an energy gap $E_{g}$. (d) PL spectra excited at $1.5 \mathrm{eV}$ for different excitation fluence. Solid lines are fits using Eq. (3). Spectra are corrected for the detection system response. Inset: fitted values of $Q_{s}$ vs fluence.

Although the mechanism should be common in condensed-matter systems, blueshifted PL of comparable brightness and bandwidth to that in graphene has never been observed. Elsaesser et al. ${ }^{15}$ probed blueshifted PL from nonthermal electrons gaining energy via intraband carrier scattering in bulk GaAs but it was marginally detectable and appeared in a very narrow frequency range. The graphene nonlinear PL is particularly efficient and broadband due to several unique properties of graphene: (1) carrier-carrier scattering requires both energy and momentum conservations, which are readily satisfied in the linear bands of graphene as illustrated in Fig. 3(b). In contrast, the phase space for the same process is much more limited in systems with nonlinearly dispersive bands [Fig. 3(c)]. (2) The linear bands lead to a symmetric distribution of nonequilibrium electrons and holes, which facilitates direct recombination of excited e-h pairs. (3) The energy acquired by an e-h pair via intraband scattering cannot be more than its kinetic energy, which equals $h \omega-E_{g}$ [Fig. 3(c)] and is the maximum in zerogap materials such as graphene. (4) The reatly reduced dielectric screening in the 2D graphene results in a very high Coulomb scattering rate ${ }^{14,16}$ that is essential for establishing the transient, highly nonequilibrium carrier population, and the strong e-h recombination. In thicker graphene films, more effective screening, ${ }^{17}$ and more attenuation of light could cause the PL to saturate.

To gain a more quantitative understanding, we modeled the process based on a simplified rate equation. Here we focus on the blueshifted PL since it is unique in graphene. The redshifted PL is more complex as the relevant carrier distributions come not only from the mechanism depicted in Fig. 3(b) but 
also from other carrier relaxation processes common for conventional materials. Let $n(E)$ denote the number density of electrons at level $E$ above the Dirac point, and $\alpha_{0} I_{0}$ be the excitation density. For $E>E_{0}$, we have

$$
\begin{aligned}
d n(E) / d t= & \gamma_{1}(E) \cdot\left(\alpha_{0} I_{0}\right)^{2}-\gamma_{2}(E) \cdot\left(\alpha_{0} I_{0}\right) \cdot n(E) \\
& -\gamma_{3}(E) \cdot n(E)
\end{aligned}
$$

The first two terms on the right-hand side of Eq. (1) describe the generation and depletion, respectively, of $n(E)$ due to scattering between excited carriers, and the last term is due to relaxation processes independent of $I_{0}$. The same relation applies to the hole density $p(E)$. Since the excitation pulse duration is much longer than the carrier lifetime, ${ }^{13}$ the timeintegrated PL signal is proportional to the peak value reached at $d n(E) / d t=d p(E) / d t=0 .{ }^{18}$ Hence the signal strength at $h \omega=2 E$ is ${ }^{19}$

$$
\left.L(\omega) \propto n(E) p(E)\right|_{\max } R_{r}(\omega) \propto\left[\frac{\gamma_{3}^{-1}(E) \gamma_{1}(E) \alpha_{0}^{2} I_{0}^{2}}{1+\gamma_{3}^{-1}(E) \gamma_{2}(E) \alpha_{0} I_{0}}\right]^{2} R_{r}(\omega)
$$

with $R_{r}(\omega)$ being the radiative recombination rate. One can see immediately from Eq. (2) that $L$ is proportional to $\sim I^{4}{ }_{0}$ at very small $I_{0}$, and approaches $\sim I^{2}$ at large $I_{0}$, which agrees with our observation of a power law changing from $\sim 3$ to 2 with increasing fluence. Fitting of the spectrum-integrated PL strength using Eq. (2) [solid curves in Fig. 2(b)] yielded a value of $\gamma_{3}^{-1} \gamma_{2}$ to be $\sim 1.5 \times \quad 10^{-12}-3 \times 10^{-12} \mathrm{~cm}^{2}$ for $E \sim 1-1.5 \mathrm{eV}$. The relaxation rate $\gamma_{3}{ }^{-1}$ is dominated by interband e-h generation and carrier-phonon scattering, and has an average value $\sim 50$ fs for $E \sim 1-1.5 \mathrm{eV} .{ }^{20}$ This yields $\left(\gamma_{2} \alpha_{0} I_{0}\right)^{-1} \sim 17-33$ fs for $\alpha_{0} I_{0} \sim 10^{12}$ $\mathrm{cm}^{-2}$, consistent with the intraband carrier scattering time within $\sim 30$ fs found in Ref. 13 at a similar excitation density.

The PL spectral profile can also be calculated using Eq. (2). To focus on the essential physics, we consider only the generation process depicted in Fig. 3(b), for the carrier density at $E_{0}$ far exceeds that at $E \neq E_{0}$ and $\mathbf{k}_{1} \| \mathbf{k}_{2}$ yields the largest overlapping integral in the scattering matrix element.14 We also approximate $\gamma_{2}(E)$ to be proportional to the number of states that a carrier at $E$ can scatter to, which grows linearly with $E$. Finally, we use a relaxation rate of $\gamma_{3}(E) \propto|E|$ from the Fermi-liquid theory ${ }^{20}$ and a radiative recombination rate $R_{r}(\omega) \propto \omega_{3}$ under the Weisskopf-Wigner approximation. ${ }^{21}$ Using results in Ref. 14 for the Coulomb scattering rate $\gamma_{1}(E)$, we then find

$$
\left.L(\omega) \propto n(E) p(E)\right|_{\max } R_{r}(\omega) \propto E^{-2} \frac{E_{0}^{2}-\left(E-E_{0}\right)^{2}}{\left[\left(E-E_{0}\right)+\hbar v_{F} Q_{s}\right]^{4}} \omega^{3}
$$

with $v_{F}$ being the Fermi velocity and $Q s$ the Thomas-Fermi screening wave vector. ${ }^{14}$ Fits of the PL spectra using Eq. (3) are shown in Fig. 3(d) with the fitted values of $Q_{s}$ being $\sim 0.06 \AA^{-1}$ [Fig. 3(d), inset], consistent with the theoretical prediction for a carrier density $\sim 10^{12} \mathrm{~cm}^{-2} \cdot{ }^{14,16}$ Hence we see this simple model provides a good qualitative description of the nonlinear PL.

To summarize, we have detected a strong, broadband nonlinear PL in graphene thin layers upon femtosecond laser irradiation. It is due to a mechanism distinct from betterknown coherent nonlinear optical processes, ${ }^{22}$ and derives its high efficiency over a wide energy range from the peculiar band structure and 2D nature of graphene. The PL has potential applications in optical labeling and imaging, and future study on a time-resolved basis shall provide insight into the interaction of highly nonequilibrium carriers in graphene at the very early relaxation stage. We thank Richard Chim for sample preparation, and Peter Yu and Dung-Hai Lee for helpful discussions. W.L. and F.W. acknowledge support from MURI-ONR. The experiment was performed at the Molecular Foundry of the Lawrence Berkeley National Laboratory supported by the DOE under Contract No. DE-AC02-05CH11231. 
1. B. Zhang, Y.-W. Tan, H. L. Stormer, and P. Kim, Nature (London)438, 201 (2005); K. Nomura and A. H. MacDonald, Phys. Rev. Lett. 96, 256602 (2006); Z. Chen, Y.-M. Lin, M. J. Rooks, and Ph. Avouris, Physica E 40, 228 (2007); A. K. Geim, Science 324, 1530 (2009).

2. Min, R. Bistritzer, J.-J. Su, and A. H. MacDonald, Phys. Rev. B 78, 121401(R) (2008).

3. Lee, X. Wei, J. W. Kysar, and J. Hone, Science 321, 385 (2008).

4. R. Nair, P. Blake, A. N. Grigorenko, K. S. Novoselov, T. J. Booth, T. Stauber, N. M. R. Peres, and A. K. Geim, Science 320, 1308 (2008); K. F. Mak, M. Y. Sfeir, Y. Wu, C. H. Lui, J. A. Misewich, and T. F. Heinz, Phys. Rev. Lett. 101, 196405 (2008).

5. J. Yan, Y. B. Zhang, P. Kim, and A. Pinczuk, Phys. Rev. Lett. 98, 166802 (2007); F. Wang, Y. B. Zhang, C. Tian, C. Girit, A. Zettl, M. Crommie, and Y. R. Shen, Science 320, 206 (2008); Z. Q. Li, E. A. Henriksen, Z. Jiang, Z. Hao, M. C. Martin, P. Kim, H. L. Stormer, and D. N. Basov, Phys. Rev. Lett. 102, 037403 (2009); Y. Zhang, T.-T. Tang, C. Girit, Z. Hao, M. C. Martin, A. Zettl, M. Crommie, Y. R. Shen, and F. Wang, Nature (London) 459, 820 (2009).

6. D. S. Chemla and D. A. B. Miller, J. Opt. Soc. Am. B 2, 1155 (1985); T. Takagahara and E. Hanamura, Phys. Rev. Lett. 56, 2533 (1986); K. B. Ferrio and D. G. Steel, Phys. Rev. B 54, R5231 (1996); U. Neukirch, S. R. Bolton, N. A. Fromer, L. J. Sham, and D. S. Chemla, Phys. Rev. Lett. 84, 2215 (2000).

7. G. T. Boyd, Z. H. Yu, and Y. R. Shen, Phys. Rev. B 33, 7923 (1986); M. R. Beversluis, A. Bouhelier, and L. Novotny, ibid. 68, 115433 (2003).

8. K. S. Novoselov, A. K. Geim, S. V. Morozov, D. Jiang, Y. Zhang, S. V. Dubonos, I. V. Grigorieva, and A. A. Firsov, Science 306, 666 (2004).

9. A. C. Ferrari, J. C. Meyer, V. Scardaci, C. Casiraghi, M. Lazzeri, F. Mauri, S. Piscanec, D. Jiang, K. S. Novoselov, S. Roth, and A. K. Geim, Phys. Rev. Lett. 97, 187401 (2006); D. Graf, F. Molitor, K. Ensslin, C. Stampfer, A. Jungen, C. Hierold, and L. Wirtz, Nano Lett. 7, 238 (2007).

10. C. Casiraghi, A. Hartschuh, E. Lidorikis, H. Qian, H. Harutyunyan, T. Gokus, K. S. Novoselov, and A. C. Ferrari, Nano Lett. 7, 2711 (2007).

11. J.-C. Diels and W. Rudolph, Ultrashort Laser Pulse Phenomena: Fundamentals, Techniques, and Applications on a Femtosecond Time Scale_Academic, New York, (1996).

12. M. Scharte, R. Porath, T. Ohms, M. Aeschlimann, J. R. Krenn, H. Ditlbacher, F. R. Aussenegg, and A. Liebsch, Appl. Phys. B 73, 305 (2001); M. Bauer, C. Wiemann, J. Lange, D. Bayer, M. Rohmer, and M. Aeschlimann, Appl. Phys. A 88, 473 (2007); M. Rohmer, F. Ghaleh, M. Aeschlimann, M. Bauer, and H. Hoevel, Eur. Phys. J. D 45, 491 (2007); D. Bayer, C. Wiemann, O. Gaier, M. Bauer, and M.

Aeschlimann, J. Nanomater. 2008, 249514.

13. J. M. Dawlaty, S. Shivaraman, M. Chandrashekhar, F. Rana, and M. G. Spencer, Appl. Phys. Lett. 92, 042116 (2008); M. Breusing, C. Ropers, and T. Elsaesser, Phys. Rev. Lett. 102, 086809 (2009).

14. F. Rana, Phys. Rev. B 76, 155431 (2007).

15. T. Elsaesser, J. Shah, L. Rota, and P. Lugli, Phys. Rev. Lett. 66, 1757 (1991).

16. E. H. Hwang and S. Das Sarma, Phys. Rev. B 75, 205418 (2007); F. Guinea, ibid. 75, 235433 (2007); W.-K. Tse, E. H. Hwang, and S. Das Sarma, Appl. Phys. Lett. 93, 023128 (2008). 
17. H. Miyazaki, S. Odaka, T. Sato, S. Tanaka, H. Goto, A. Kanda, K. Tsukagoshi, Y. Ootuka, and Y. Aoyagi, Appl. Phys. Express 1, 034007 (2008).

18. T. C. O’Haver and J. D. Winefordner, Anal. Chem. 38, 1258 (1966).

19. P. Y. Yu and M. Cardona, Fundamentals of Semiconductors

_Springer, New York, 1996_.

20. C. D. Spataru, M. A. Cazalilla, A. Rubio, L. X. Benedict, P. M. Echenique, and S. G. Louie, Phys. Rev. Lett. 87, 246405 (2001).

21. W. Weisskopf and E. Wigner, Z. Phys. 63, 54 (1930).

22. See, for example, D. Sun, C. Divin, J. Rioux, J. E. Sipe, C. Berger, W. A. de Heer, P. N. First, and T. B. Norris, Nano Lett. 10, 1293 (2010). 


\section{LEGAL DISCLAIMER}

This document was prepared as an account of work sponsored by the United States Government. While this document is believed to contain correct information, neither the United States Government nor any agency thereof, nor The Regents of the University of California, nor any of their employees, makes any warranty, express or implied, or assumes any legal responsibility for the accuracy, completeness, or usefulness of any information, apparatus, product, or process disclosed, or represents that its use would not infringe privately owned rights. Reference herein to any specific commercial product, process, or service by its trade name, trademark, manufacturer, or otherwise, does not necessarily constitute or imply its endorsement, recommendation, or favoring by the United States Government or any agency thereof, or The Regents of the University of California. The views and opinions of authors expressed herein do not necessarily state or reflect those of the United States Government or any agency thereof or The Regents of the University of California. 